\title{
Manejo quirúrgico de prolapso uretral en pediatría: Reporte de caso
}

\author{
Surgical management of urethral prolapse in pediatrics: A case report \\ J. Cristina Guerrero-Villota1', Manuel F. Dueñas-Dasilva'1, Juan Camilo Polania-Quintero', \\ Luis Mauricio Figueroa-Gutiérrez² $\mathbb{D}$, Juan Carlos Dueñas-Ramírez² (D) \\ 1 Médico, Pontificia Universidad Javeriana, Cali, Colombia. \\ 2 Médico, especialista en Cirugía Pediátrica, Sección de Cirugía Pediátrica, Universidad del Valle, Cali, Colombia.
}

\section{Resumen}

Introducción. El prolapso uretral es una entidad poco común, con una incidencia estimada de 1 en 3000 mujeres. Se presenta cuando la mucosa uretral sobresale espontáneamente más allá del meato uretral. Es una patología poco diagnosticada dada su baja frecuencia y de allí la importancia de conocer sobre su presentación, diagnóstico y tratamiento. El objetivo de este artículo fue presentar el caso de una paciente de 10 años con diagnóstico de prolapso uretral y su manejo quirúrgico.

Caso clínico. Paciente femenina de 10 años, que consultó por cuadro clínico de 1 año de evolución consistente en dolor en región urogenital, que se irradiaba a hipogastrio, asociado a pujo y disuria, a quien se le diagnosticó prolapso uretral y se realizó corrección quirúrgica de mucosa uretral prolapsada mediante técnica de Kelly-Burnham modificada.

Conclusión. El prolapso uretral es una entidad que con frecuencia es diagnosticada erróneamente pues su diagnóstico es eminentemente clínico. Si bien se ha descrito el tratamiento médico en primera instancia, éste tiene una alta tasa de recurrencia, por lo que en estos casos se prefiere la resección quirúrgica del tejido prolapsado.

Palabras clave: uretra; prolapso; pediatría; tratamiento conservador; cirugía.

\begin{abstract}
Introduction. Urethral prolapse is a rare entity, with an estimated incidence of 1 in 3,000 women. It occurs when the urethral mucosa spontaneously protrudes beyond the urethral meatus. It is a poorly diagnosed pathology given its low frequency and hence the importance of knowing about its presentation, diagnosis and treatment. The objective of this article was to present the case of a 10-year-old patient with a diagnosis of urethral prolapse and its surgical management.
\end{abstract}

Fecha de recibido: 07/01/2021 - Fecha de aceptación: 16/03/2021 - Publicación en línea: 17/09/2021

Correspondencia: J. Cristina Guerrero-Villota, Calle 5a \# 60 - 64, Unidad Asturias, Apartamento 502B, Cali, Colombia.

Teléfono: 3116648303. Correo electrónico: cristina.guerrero.villo@gmail.com

Citar como: Guerrero-Villota JC, Dueñas-Dasilva MF, Polania-Quintero JC, Figueroa-Gutiérrez LM, Dueñas-Ramirez JC. Manejo quirúrgico de prolapso uretral en pediatría: reporte de caso. Rev Colomb Cir. 2022;37:156-61. https://doi.org/10.30944/20117582.870

Este es un artículo de acceso abierto bajo una Licencia Creative Commons - BY-NC-ND https://creativecommons.org/licenses/by-nc-nd/4.0/deed.es 
Clinical case. A 10-year-old female patient, who consulted for a 1-year clinical picture consisting of pain in the urogenital region, radiating to the hypogastrium, associated with pushing and dysuria, who was diagnosed with urethral prolapse and a surgical correction of the urethral mucosa was performed prolapsed by modified Kelly-Burnham technique.

Conclusion. Urethral prolapse is an entity that is frequently misdiagnosed because its diagnosis is eminently clinical. Although medical treatment has been described in the first instance, it has a high recurrence rate, so surgical resection of the prolapsed tissue is preferred in these cases.

Keywords: urethra; prolapse; pediatrics; conservative treatment; surgery.

\section{Introducción}

El prolapso uretral (PU) es una entidad poco común, que se presenta cuando la mucosa de la uretra sobresale espontáneamente más allá del meato uretral ${ }^{1,2}$, lo que resulta en congestión vascular y edema del tejido prolapsado ${ }^{2}$. El prolapso uretral en la población pediátrica suele presentarse con mayor frecuencia de manera asintomática ${ }^{3,4}$ y cuando causa sintomatología, la hemorragia urogenital es la principal manifestación ${ }^{5}$.

\section{Caso clínico}

Se trata de una paciente de 10 años que consultó por cuadro clínico de un año de evolución consistente en dolor en región urogenital, que se irradiaba a hipogastrio, asociado a pujo y disuria; negaba sangrado urogenital u otros síntomas. El cuadro se exacerbó en los últimos 8 días, por lo cual consultó. Como antecedentes relevantes presentaba historia de larga data de estreñimiento, con presencia de fecaloma palpable al ingreso, por lo cual requirió manejo médico con polietilenglicol, y como antecedente familiar, una hermana de 15 años con diagnóstico de prolapso uretral corregido quirúrgicamente.

Al examen físico se observaba una pequeña masa que ocluía parcialmente el introito (Figura 1) y a la tracción de los labios mayores se observaba protrusión de mucosa por fuera del meato uretral (Figura 2).

Se decidió iniciar manejo conservador con estrógenos conjugados tópicos cada 8 horas hasta completar 7 días. Al término del tratamiento la paciente negó mejoría en la sintomatología, por lo que se decidió programar para corrección quirúrgica del prolapso uretral.

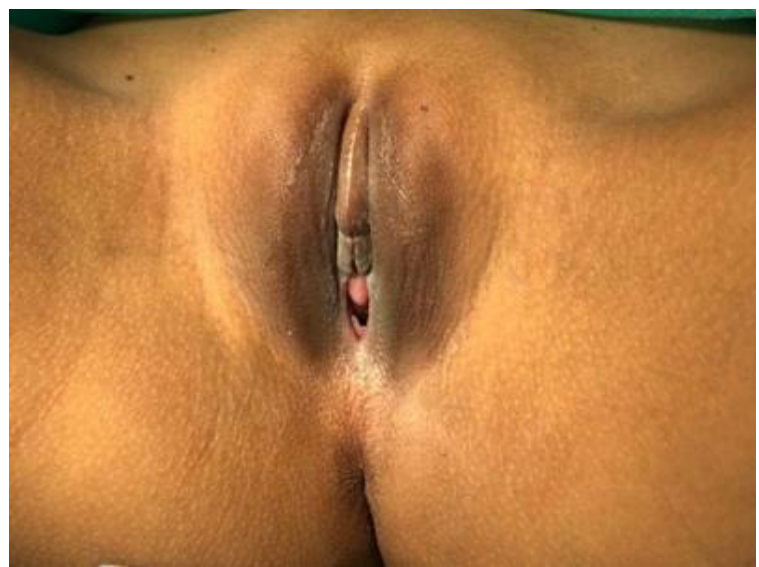

Figura 1. Examen genitourinario inicial donde se observa masa que ocluye parcialmente el introito y mucosa uretral prolapsada.

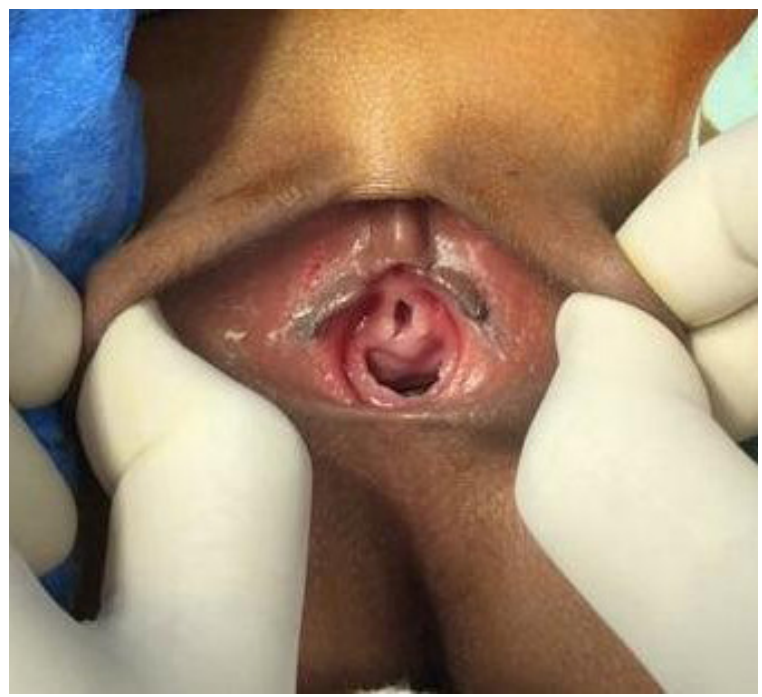

Figura 2. Tracción de los labios mayores donde se aprecia protrusión de mucosa por fuera del meato uretral. 


\section{Procedimiento quirúrgico}

Se utilizó la técnica de Kelly-Burnham modificada, o escisión de cuatro cuadrantes ${ }^{2,3}$. Se ubicó la paciente en posición de litotomía, y a la tracción de los labios mayores se observó el prolapso circunferencial de la mucosa de la uretra de aproximadamente $1 \mathrm{~cm}$. Se procedió a colocar cuatro suturas de reparo de seda 3-0 en las posiciones 12, 3, 6, y 9 horas (Figura 3). Posteriormente se llevó a cabo la resección por cuadrantes de mucosa prolapsada y finalmente se realizó la aproximación inmediata de la unión mucocutánea con sutura absorbible 4-0 (Figura 4). La evolución postquirúrgica de la paciente durante las primeras semanas fue satisfactoria.

\section{Discusión}

El prolapso uretral fue descrito por primera vez en 1732 por Solingen ${ }^{2,6}$. Es una entidad poco común, con una incidencia estimada de 1 en 3000 mujeres ${ }^{2,4}$. Esta afección se observa como una protuberancia circular, en forma de dona, que puede medir entre $0,5 \mathrm{~cm}$ y $3 \mathrm{~cm}$ de diámetro ${ }^{1,2}$. Una vez la mucosa uretral se prolapsa a través del meato uretral éste genera contracción de forma circunferencial debido al tono muscular propio del meato, lo que produce diversos grados de edema, congestión vascular y necrosis ${ }^{4,7}$.

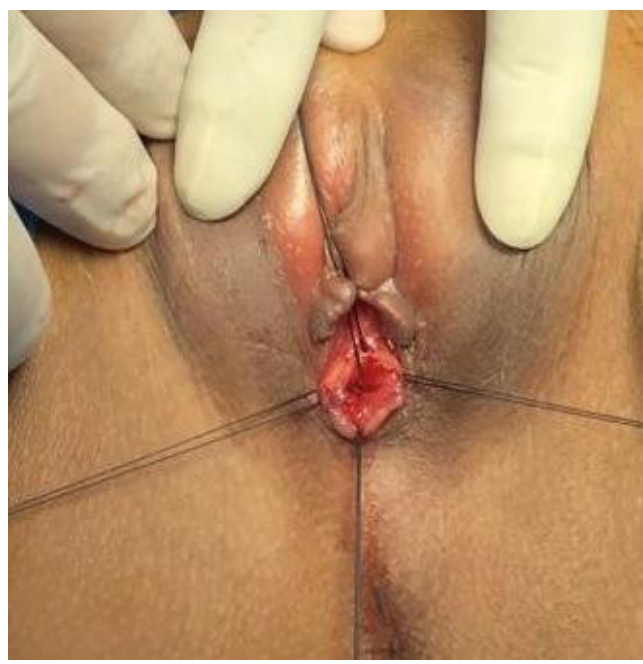

Figura 3. Ubicación de suturas de reparo con seda 3-0 en las posiciones $12,3,6$, y 9 horas.
Esta rara condición se presenta con mayor frecuencia en niñas prepúberes y en mujeres posmenopáusicas ${ }^{2}$. Se ve más comúnmente en niñas afrodescendientes y rara vez se observa en las niñas caucásicas ${ }^{7-10}$. Los casos descritos en pediatría van de los 18 meses a los 10 años de edad, con 2 picos entre los 3 a 5 años y 5 a 9 años ${ }^{9,10}$. Debido a la baja frecuencia de esta patología, la tasa de diagnósticos erróneos es alta ${ }^{4,6}$.

La fisiopatología del PU sigue siendo incierta. Se han propuesto diversas causas, entre ellas, malformaciones o estructuras de colágeno débiles ${ }^{3}$, lo que conlleva a la presencia de un tejido de soporte periuretral inadecuado, con debilidad del tejido submucoso, que finalmente se observa como una mala unión entre la mucosa uretral y el músculo subyacente ${ }^{7,9}$. Esta hipótesis se ha visto reforzada dado que muchos pacientes refieren antecedentes familiares de prolapso uretrales y vaginales. En el artículo de Fornari et al. ${ }^{3}$ se comenta de un caso de una madre e hija con prolapso uretral. En el caso aquí descrito la paciente tenía una hermana de 15 años que requirió corrección quirúrgica del prolapso uretral.

Se sugieren otras posibles etiologías, como el aumento de la presión intraabdominal, la disfunción neuromuscular y una redundancia excesiva de la mucosa uretral ${ }^{8,9}$. Sin embargo, la principal hipótesis etiológica es la deficiencia de estrógenos, dado la preponderancia del prolapso uretral en el periodo prepuberal y posmenopáusico ${ }^{2,3}$.

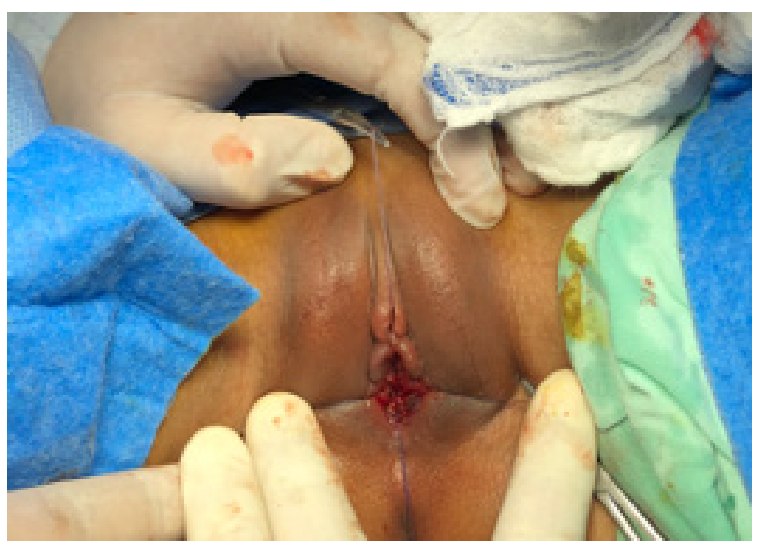

Figura 4. Técnica de Kelly Burnham modificada o escisión de cuatro cuadrantes. 
También se han descrito en la literatura factores de riesgo para PU como tos crónica y estreñimiento ${ }^{6,7}$, dado que estos causan aumento de la presión intraabdominal. En nuestro caso la paciente tenía historia de estreñimiento de larga data y tratamiento médico para su condición, sin embargo, se requieren estudios con series más grandes para poder establecer la relación entre estreñimiento y PU. La relación entre trauma genital y PU aún es controvertida ${ }^{7}$.

El PU en la población pediátrica suele presentarse con mayor frecuencia de manera asintomática ${ }^{2,4}$ y el motivo de consulta es una masa redondeada, lisa y rojiza que observa el cuidador ${ }^{6,7}$. Cuando el PU causa síntomas, principalmente se manifiesta como hemorragia en la región urogenital ${ }^{2,7,8,10}$, que puede presentarse en diversos grados, desde manchas de sangre en la ropa interior hasta sangrado franco en el área. Raras veces se acompaña de otros síntomas como disuria, pujo, retención urinaria, polaquiuria o dolor introital ${ }^{1,4,10}$. En el caso reportado la principal sintomatología de la paciente fue dolor en región urogenital, que se irradiaba a hipogastrio, asociado a pujo y disuria; sin embargo, referían que su hermana había presentado hemorragia urogenital franca.

El diagnóstico del PU es clínico ${ }^{2,6,7}$ por lo que es importante obtener una historia clínica completa y un adecuado examen físico, en el que sea posible descartar otras patologías ${ }^{1}$. Ante la presencia de hemorragia urogenital se debe realizar un examen ginecológico completo, el cual para llevarse a cabo de manera adecuada podría requerir inspección bajo anestesia general ${ }^{1}$. En la evaluación, la mucosa prolapsada aparece como una masa de tejido congestionado que rodea al meato uretral ${ }^{10}$, donde es importante identificar el meato uretral en el centro del tejido edematoso. En caso de que la congestión y el edema sean severos, se puede hacer uso de una sonda de alimentación 8 Fr o una sonda Foley para localizar el meato, y se considera que con esta maniobra es suficiente para establecer el diagnóstico ${ }^{4,5}$.

Dada la baja experiencia con este tipo de patología, es muy fácil confundirla con otras entidades y retrasar el tratamiento, por lo que es importante conocer los diagnósticos diferenciales, entre los cuales están el papiloma uretral, carúncula, pólipo, ureterocele prolapsado, rabdomiosarcoma vaginal, himen imperforado y abuso sexual ${ }^{10,11}$.

En caso de ureterocele, éste sobresale en forma asimétrica y no circunferencial como se mencionó anteriormente. Si se desconoce el prolapso uretral, es común que la apariencia de la lesión lleve a una mala interpretación de abuso sexual, tal como lo describen Montes et al., quienes reportan el caso de una paciente de 23 meses de edad con historia de sangrado vaginal, con sospecha de abuso sexual y matrato infantil, que después de un riguroso examen bajo sedación se logró hacer el diagnostico de prolapso uretral ${ }^{12}$.

Como se cree que los niveles bajos de estrógenos podrían estar detrás de la fisiopatología del prolapso uretral, se ha propuesto el tratamiento médico consistente en el uso de cremas con estrógenos conjugados en la zona urogenital y baños de asiento ${ }^{1,2,4}$, con el objetivo de disminuir la inflamación y el edema. En muchos casos se propone este tratamiento como el manejo inicial, sin embargo la tasa de éxito es muy variable, oscilando entre el 33 al $92 \%{ }^{3}$. Además, tiene la limitación de presentar altas tasas de recurrencia, reportadas hasta en el $67 \%$ de los $\operatorname{casos}^{6,10}$.

Cuando el prolapso uretral no mejora con el tratamiento médico es necesaria la resección quirúrgica de los bordes de mucosa ${ }^{2,5,7}$, como lo describen Martínez et al. ${ }^{9}$.

La extirpación quirúrgica de la mucosa uretral se ha asociado con tasas de curación más altas, un alivio rápido de los síntomas y una estancia hospitalaria más corta ${ }^{2,4,10}$. El procedimiento se realiza en el quirófano bajo anestesia general. Se han descrito varias técnicas entre las cuales se encuentran la ligadura de la mucosa alrededor de una sonda (con la que se ha obtenido una alta incidencia de dolor postoperatorio), la cauterización de la mucosa y la escisión de la mucosa con sonda in situ ${ }^{5,7}$, siendo esta la más popular. Sin embargo, tiene dificultades técnicas pues realizar la incisión alrededor de una sonda en un área tan confinada puede ser muy engorroso y no hay nada que evite que la mucosa 
proximal se retraiga, y si esto ocurre es más difícil realizar la anastomosis mucocutánea, aumentando el riesgo de estenosis del meato ${ }^{5}$.

Otra técnica descrita y ampliamente utilizada es la técnica de Kelly-Burnham modificada o escisión de cuatro cuadrantes, siendo ésta la que se eligió para la corrección del PU de la paciente. Esta técnica evita la colocación de una sonda en el meato mientras se realiza la cirugía y, por lo tanto, reduce el riesgo de retracción de la mucosa proximal. Se colocan cuatro suturas de sujeción en las posiciones 12, 3, 6, y 9 horas; la sutura de sujeción evita la retracción de la mucosa proximal, que se ve más fácilmente sin la sonda in situ. Una vez colocadas las suturas de sujeción se realiza la escisión por cuadrantes, seguida de la anastomosis de la unión mucocutánea con suturas absorbibles.

En la publicación de Jerkins et al. ${ }^{10}$, en donde se reporta el uso de 6 enfoques de tratamiento para comparar la tasa de complicaciones, la tasa de recurrencia y la duración de la estancia hospitalaria de cada una de ellas, los resultados evidencian que, de 40 pacientes con prolapso uretral, a 15 niñas se les realizó escisión primaria de la mucosa prolapsada y no presentaron complicaciones ni recidivas y la estancia hospitalaria fue de un día.

Con esta y otras publicaciones ${ }^{9}$ se evidencia que la escisión quirúrgica de la mucosa es una buena opción de manejo en pacientes con recurrencias o en quienes el manejo médico no ha sido efectivo.

\section{Conclusión}

El prolapso uretral es una entidad poco común, que aparece más frecuentemente en niñas prepúberes, y más comúnmente en niñas afrodescendientes. Debido a la baja frecuencia de esta patología, la tasa de diagnósticos erróneos es alta, siendo importante el conocimiento de la misma.

El diagnóstico del PU es clínico, se sospecha en niñas con antecedente de hemorragia urogenital. Para descartar otros diagnósticos es preciso realizar un adecuado examen de la zona genitourinaria, donde se demuestre la mucosa prolapsada que rodea al meato uretral, o sea que el meato se encuentra en el centro del tejido edematoso y que éste lo rodea circunferencialmente.

Si bien se ha descrito el manejo médico como primera línea, éste tiene una alta tasa de recurrencia, por lo que, según los datos obtenidos de la revisión de la literatura y la experiencia en este caso, se prefiere la resección quirúrgica del tejido prolapsado, la cual se ha asociado a un alivio rápido de los síntomas y una menor tasa de recidiva.

Agradecimientos: A las directivas del Hospital Universitario del Valle "Evaristo García" E.S.E. por darnos la oportunidad de desarrollar nuestra práctica en cirugía pediátrica en sus instalaciones y a su comité de ética en Investigaciones por aprobar el diseño y contenido del artículo.

\section{Cumplimiento de normas éticas}

Consentimiento informado. Se obtuvo el consentimiento informado por parte de la madre de la paciente para las intervenciones quirúrgicas, la participación en publicaciones y la toma de fotografías. El comité de ética institucional aprobó el diseño y el contenido del artículo.

Declaración de conflicto de intereses. Los autores declaran que no tienen conflicto de intereses.

Fuentes de financiación: recursos propios de los autores.

\section{Contribución de los autores:}

Concepción y diseño del estudio: J. Cristina GuerreroVillota, Manuel F. Dueñas-Dasilva, Juan Camilo Polania-Quintero, Luis Mauricio Figueroa, Juan Carlos Dueñas-Ramirez.

Evaluación de la paciente y examen físico: Juan Carlos Dueñas-Ramirez.

Adquisición de datos, análisis e interpretación de datos: J. Cristina Guerrero-Villota, Manuel F. Dueñas-Dasilva, Juan Camilo Polania-Quintero, Luis Mauricio Figueroa, Juan Carlos Dueñas-Ramirez.

Redacción del manuscrito: J. Cristina Guerrero-Villota, Manuel F. Dueñas-Dasilva, Juan Camilo Polania-Quintero, Luis Mauricio Figueroa, Juan Carlos Dueñas-Ramirez.

Revisión crítica: J. Cristina Guerrero-Villota, Manuel F. Dueñas-Dasilva, Juan Camilo Polania-Quintero, Luis Mauricio Figueroa, Juan Carlos Dueñas-Ramirez. 


\section{Referencias}

1. Vunda A, Vandertuin L, Gervaix A. Urethral prolapse: An overlooked diagnosis of urogenital bleeding in premenarcheal girls. J Pediatr. 2011;158:682-3. https://doi.org/10.1016/j.jpeds.2010.11.050

2. Olumide A, Olusegun AK, Babatola B. Urethral mucosa prolapse in an 18-year-old adolescent. Case Rep Obstet Gynecol. 2013;2013:1-3. https://doi.org/10.1155/2013/231709

3. Fornari A, Gressler M, Murari JCL. Urethral prolapse: A case series and literature review. J Obstet Gynaecol India. 2020;70:158-62. https://doi.org/10.1007/s13224-019-01288-2

4. Igwebueze OI, Asimadu EE. Premenarcheal urethral mucosa prolapse in Enugu, Southeast, Nigeria: Five case series. J Women's Health Care. 2015;4:4-6. https://doi.org/10.4172/2167-0420.1000243

5. Shurtleff BT, Barone JG. Urethral prolapse: Four quadrant excisional technique. J Pediatr Adolesc Gynecol. 2002;15:209-11.

https://doi.org/10.1016/s1083-3188(02)00157-2

6. Wei Y, Wu S, Lin T, He D, Li X, Wei G. Diagnosis and treatment of urethral prolapse in children: 16 years' experience with 89 Chinese girls. Arab J Urol. 2017;15:248-53.

https://doi.org/10.1016/j.aju.2017.03.004

7. Zager RP. Urethral prolapse in young girls. AMA J Dis Child. 1957;94:196-9. https://doi.org/10.1001/archpedi.1957.04030030090014

8. Lowe FC, Hill GS, Jeffs RD, Brendler CB. Urethral prolapse in children: Insights into etiology and management. J Urol. 1986;135:100-3. https://doi.org/10.1016/s0022-5347(17)45530-3

9. Martinez-Casas A, Rosales-Morán AP. Prolapso parcial de mucosa uretral en niñas. Revisión de dos casos clínicos y propuesta de un nuevo abordaje quirúrgico. Bol Med Hosp Infant Mex. 2010;67:58-62.

10. Jerkins GR, Verheeck K, Noe HN. Treatment of girls with urethral prolapse. J Urol. 1984;132:732-3. https://doi.org/10.1016/s0022-5347(17)49845-4

11. Lago-Barney G, Cespedes-Londoño JA. Abuso sexual infantil. Prescop SCP 3. 2006;16-30.

12. Montes GA, García HA, Pataquiva ME. Prolapso uretral: Un diagnóstico diferencial del abuso sexual en niños. Cuad Med Forense. 2009; 58:289-92. 